\title{
The Effect of Niclosamide on Certain Biological and Biochemical Properties of Drosophila melanogaster
}

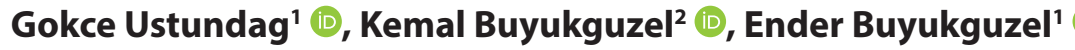 \\ 'Zonguldak Bülent Ecevit University, Faculty of Arts and Sciences, Department of Molecular Biology and Genetics, Zonguldak, Turkey \\ ZZonguldak Bülent Ecevit University, Faculty of Arts and Sciences, Department of Biology, Zonguldak, Turkey
}

ORCID IDs of the authors: G.U. 0000-0002-4026-1358; K.B. 0000-0002-4442-5081; E.B. 0000-0002-6959-8480

Please cite this article as: Ustundag G, Buyukguzel K, Buyukguzel E. The Effect of Niclosamide on Certain Biological and Biochemical Properties of Drosophila melanogaster. Eur J Biol 2019; 78(1): 29-39.

\section{ABSTRACT}

Objective: Laboratory research on management strategies such as biological and chemical control usually demands rearing high numbers of high-quality insects. For this reason, there is a need for detailed information about which antimicrobial agents should be added to their diets in order to obtain high quality insects.

Materials and Methods: Niclosamide, which is an anthelmintic derivative of salisilanilid, was added in amounts of 100 , 200, 400 and $800 \mathrm{mg} / \mathrm{L}$ into artificial diets of Drosophila melanogaster (Meigen). The effects on survival rate, developmental time and adult longevity of the insects were examined. Furthermore, the effect of this anthelmintic antibiotic on important oxidative stress indicators; lipid peroxidation product, malondialdehyde (MDA) and protein oxidation products; protein carbonyl (PCO) contents and a detoxification enzyme, glutathione S-transferase (GST) activity in 3rd instar larvae, pupae and adult stage of the insect were also investigated.

Results: Compared with those insects on the control diet, the tested concentrations of niclosamide significantly decreased survival rate in all developmental stages of the insect. The control diet produced $94.0 \pm 1.0 \%$ of $3^{\text {rd }}$ stage larvae whereas in the highest concentration ( $800 \mathrm{mg} / \mathrm{L}$ ) this ratio decreased to $14.00 \% \pm 1.73$. While $42.08 \pm 0.50 \%$ of the adults survived in the control diet, longevity decreased to $2.30 \pm 0.15$ days in the highest concentration. All concentrations of niclosamide resulted in increased MDA contents in the last larval stage of $D$. melanogaster and concentrations of 100,200 and $800 \mathrm{mg} / \mathrm{L}$ niclosamide increased pupal MDA content in comparison to the control group. At low concentrations of niclosamide, PCO decreased in the last stage of larvae. The diet with $400 \mathrm{mg} / \mathrm{L}$ niclosamide concentration significiantly increased GST activity in pupal stage.

Conclusion: The results of this work indicated that the negative effects of niclosamide on biological characteristics of $D$. melanogaster are due to an increase in the oxidative stress and crippled detoxification capacity of the insect.

Keywords: Drosophila melanogaster, oxidative stress, niclosamide, survival, development

\section{INTRODUCTION}

Agricultural pesticides that reduce the growth and yield of plants of nutritional significance are widely used today. Although these pesticides are effective methods for controlling insect populations in agricultural fields the improper use of these pesticides can cause several pest species to develop resistance against them (1).

Recent studies have led to the use of clinically important antimicrobial antibiotics as pesticides and significant results were obtained in these studies. In previous studies, antibiotics were used to prevent microbial contamination, to stimulate food intake, and to determine their effects on pests by administering them into the culture medium where pests were propagated (2-8). The most recent studies have demonstrated that these antibiotics cause oxidative effects on pests in various biochemical assays. Büyükgüzel and Kalender (9-11) reported that changes in the activities of alanine aminotransferase and aspartate aminotransferase enzymes are indicators of the severity of antibiotic 
toxicity in pests. In many studies, it has been shown that a low concentration of antibiotics can be used to protect the synthetic diets from contamination and also that high concentrations of antibiotics can be used in insect management (8-15).

Furthermore, it is also known that certain insects, including Galleria mellonella (Linnaeus), Drosophila melanogaster (Meigen) are used as a models to test the action of clinical antiparasitic, antibacterial and antifungal drugs (16). Several species in the Drosophila genus became model organisms used in genetic and biochemical studies due to their easy culture in a laboratory environment, short life cycle, and the ability to produce a large number of offspring in one generation (17). Thus, it is a suitable experimental animal to test the biological effects of certain commonly used chemicals (18-20).

Niclosamide is among the active ingredients of drugs used in the treatment of infections induced by cestodes in humans and animals $(21,22)$. Niclosamides act by inhibiting oxidative phosphorylation in parasite mitochondria. Furthermore, they can inhibit the anaerobic metabolism in several helminthic parasites. Anthelmintic drugs interfere with neuromuscular transmission by disrupting the main energy metabolism pathways in parasites. By affecting glucose absorption or transportation, they disrupt the glycogen metabolism, preventing glycolysis. They inhibit nucleic acid synthesis and eventually reproduction. Niclosamide is not significantly absorbed in the gastrointestinal tract and excreted in the stool. The absorbed part is converted to an active metabolite, aminoniclosamide (23).

Various xenobiotics stimulate production of free radicals (24) which lead to oxidative damage in all cellular compounds (carbohydrate, lipid, protein, DNA, RNA), especially to membrane phospholipids. As a result, the membranes become depolarized, the activity of the detoxifying enzymes increases, and the permeability of the cell membrane and the electrical charge balance changes (25). Antioxidants are vital biomolecules that can dissolve in water or oil, providing defense against free radicals and oxidative stress that are harmful to the body (26). Antioxidant enzymes are capable of minimizing the damage caused by free radicals, or eliminating it. These enzymes are catalase (CAT), glutathione-S-transferases (GST), peroxidase, and superoxide dismutase (SOD). GSTs are detoxifying enzymes found in vertebrates, plants, insects, yeasts, and aerobic bacteria (27). The phase II biotransformation enzyme GST catalyzes the conjugation of glutathione to a wide variety of xenobiotics with an electrophilic site, yielding more water-soluble xenobiotics and facilitating their excretion. Therefore, we decided to examine if niclosamide can alter lipid peroxidation, measured as malondialdehyde (MDA) concentration, as well as the activity of the detoxification enzyme GST.

In the present study, D. melanogaster was used as a model organism. The effects of niclosamide (a salicylanilide group anthelminthic substance) on the biological properties of $D$. melanagaster, lipid peroxidation and protein oxidation levels in the $3^{\text {rd }}$ stage larval, pupal and adult stages of the insect and detoxification enzyme GST activity and the biochemical and physiological bases of the correlation between the oxidative stress and the mechanism of action of certain concentrations of niclosamide on biological properties of the insect were investigated.

\section{MATERIALS AND METHODS}

Drosophila melanogaster Culture and Experimental Medium $W^{1118}$ strain adult D. melanogaster (Diptera: Drosophilidae) individuals were procured from the University of Masaryk, Czech Republic, and brought to our laboratory. Stock culture was produced in the Zonguldak Bülent Ecevit University, Molecular Biology and Genetics Department, Biochemistry and Physiology research laboratory in glass bottles with artificial diets. The egglaying of adult individuals, larval stage, pupal stage and adult stage were sustained on this artificial diet including potatoes and sucrose $(28,29)$ and the adult individuals were sustained with a new culture. The stock culture and experimental setup were conducted in a laboratory environment with a relative humidity of $60-70 \%, 12$ hours of light, 12 hours of darkness and $25 \pm 2{ }^{\circ} \mathrm{C}$. The diet used to sustain the culture was also used as a control diet in culture tests conducted to examine the effect of niclosamide on D. melanogaster.

\section{Niclosamide Use in the Experiments}

Niclosamide (2',5-Dichloro-4'-nitrosalicylanilide white yellow powder, 98\%) was obtained from Sigma-Aldrich (St. Louis, Mo., USA). Pre-nutritional tests were conducted with niclosamide to determine the range of test concentrations. The concentration range where $D$. melanogaster could complete the development stages until the adult stage was determined. In addition to the control group, 100, 200, 400 and $800 \mathrm{mg}$ niclosamide doses were added to $1000 \mathrm{~mL}$ diet in tests conducted with a niclosamide supplement. Niclosamide was added when the temperature of the food prepared for the experiment reaches room temperature. Niclosamide was mixed with the diet until it was homogenously mixed. In control experiments, only diets without niclosamide were used.

\section{Niclosamide Tests on Survival Rate and Developmental Time of $\boldsymbol{D}$. melanogaster}

In tests conducted to determine the survival rate and developmental time of $D$. melanogaster, an artificial culture medium was prepared and the tested concentrations of niclosamide were added to the culture media. Cultures were distributed in equal amounts into $15 \mathrm{ml}$ glass bottles. Twentyfive $1^{\text {st }}$ stage larvae were placed in each control media without niclosamide and the media containing 100, 200, 400 and 800 $\mathrm{mg} / \mathrm{L}$ niclosamide using a soft-tipped brush and then covered with a cotton wad. The rates of the larvae that reached to the $3^{\text {rd }}$ stage, pupa and adult stages from the $1^{\text {st }}$ stage were calculated. The time it took the larvae to reach the $3^{\text {rd }}$ stage, pupa and adult stages was determined in days. All experiments were conducted in 4 replicates at $25 \pm 2{ }^{\circ} \mathrm{C}$ and $60-70 \%$ relative humidity, 12 hours light, 12 hours dark in the laboratory. 
Niclosamide Tests on the Adult Longevity of $D$. melanogaster A $5 \mathrm{ml}$ control group culture media without niclosamide and 100, 200, 400 and $800 \mathrm{mg} / \mathrm{L}$ niclosamide administered groups were added in twenty five $15 \mathrm{ml}$ glass bottles. Using a softtipped brush, the first stage larvae were placed in the culture media. The $1^{\text {st }}$ stage larvae were cultured until the adult stage in this way. Until the adult stage, individuals were transferred daily to bottles containing $5 \mathrm{ml}$ evenly distributed culture media. The adults in the control group and the study groups with different niclosamide concentrations were checked every day at a certain time. The adult longevity of each adult was determined until the last individual expired. This process was conducted under the environmental conditions where the stock insects were cultured.

\section{Biochemical Analysis}

Twenty-five larvae, pupa and adults were collected from the $D$. melanogaster eggs released into the culture media in the control and each of the above-mentioned niclosamide concentration groups. The process was repeated four times. MDA and protein carbonyl (PCO) amounts and GST activity were determined for the collected larvae, pupae and adults. The individuals harvested at each developmental stage were extracted in a homogenization buffer using an ultrasonic homogenizer (10 sec, 30 W) (Bandelin Sonoplus, HD2070, Berlin, Germany) and conducted at $+4^{\circ} \mathrm{C}$ for 15 seconds. The obtained homogenate was centrifuged at $16000 \times \mathrm{g}$ for $20 \mathrm{~min}$ for the GST assay. In the MDA quantity assays, the samples were passed through an ultrasonic homogenizer and the homogenate was centrifuged at $2000 \mathrm{xg}$ for $15 \mathrm{~min}$ at $4{ }^{\circ} \mathrm{C}$. For the determination of PCO, the supernatant was obtained by centrifuging the homogenized samples at $1000 \times \mathrm{g}$ for $10 \mathrm{~min}$ at $+4{ }^{\circ} \mathrm{C}$. In this stage of the study, MDA, PCO levels and GST activity were determined for the larvae, pupa and adult individuals cultured in 100, 200, 400 and $800 \mathrm{mg} / \mathrm{L}$ niclosamide concentrations.

\section{Measurement of MDA Level}

An ultrasonic homogenizer was used for the disintegration of the D. melanogaster samples (15 sec, $30 \mathrm{~W}$ ) (Bandelin Sonoplus, HD2070, Berlin, Germany). The MDA level, which is the final product of lipid peroxidation with TBA, was calculated by reading the absorbance at $532 \mathrm{~nm}$. Plastic $1.5 \mathrm{~cm}$ cuvettes were used in MDA analysis. The MDA level was expressed as nmol/mg protein (30) using $1.56 \times 105 \mathrm{M}^{-1} \mathrm{~cm}^{-1}$ coefficient.

\section{Measurement of PCO Level}

Protein carbonyl determination was conducted based on the method developed by Levine et al.(31) with certain modifications (32) where with carbonyl groups in the protein formed a stable 2,4-dinitrophenyl hydrosol (DNP) with 2,4-dinitrophenyl hydrazine (DNPH) in strong acidic environment $(2 \mathrm{M} \mathrm{HCl})$ and the absorbance of the products was measured at $370 \mathrm{~nm}$.

\section{Measurement of GST Activity}

The method developed by Habig et al. (33) was used to measure the GST (EC 2.5.1.18) activity. Enzyme activity was measured as the amount of thioether generated at 1 minute per $1 \mathrm{mg}$ total protein in the supernatant at $340 \mathrm{~nm}\left(\varepsilon_{340}: 0.0096 \mu \mathrm{M} . \mathrm{cm}^{-1}\right)$. The specific activity of the enzyme is $\mu \mathrm{mol} / \mathrm{mg}$ protein $/ \mathrm{min}$.

\section{Measurement of Total Protein Content}

Total protein amounts of the samples were used to calculate MDA levels and GST activity. The absorbances of the samples were measured at $600 \mathrm{~nm}$. Different concentrations of BSA (Bovine serum albumin) solutions were prepared for the protein assay and a standard graph was obtained. Total protein content was calculated using the above-mentioned standard graph (34). Total protein assay was conducted to calculate the PCO level that occurred as a result of protein oxidation. The absorbance of the samples was measured spectrophotometrically at 280 $\mathrm{nm}$. BSA standard solutions were prepared with $6 \mathrm{M}$ guanidine hydrochloride. The total protein amount was calculated by plotting a standard graph (35).

\section{Statistical Analysis}

One-way analysis of variance (ANOVA) was used to analyze the data on the developmental time, adult longevity, MDA, PCO levels, GST activities (36), the "LSD Test" (37) was used to determine the significance of the difference between the means, and the "X2 (Chi square) test" was used to analyze the survival rate (36). The significance of the mean values was determined at a 0.05 significance level. Correlation analysis was also performed between the tested concentrations and our data (37).

\section{RESULTS}

\section{The Effects of Niclosamide on Survival Rate and Developmental Time of $D$. melanogaster Larvae}

The survival rate of $D$. melanogaster indicated that all tested niclosamide concentrations significantly reduced the survival rate of $3^{\text {rd }}$ stage larvae, pupae and adults when compared to the niclosamide-free control diet (Figures 1-3). We found a significant negative correlation between the niclosamide concentration and survival rate of $3^{\text {rd }}$ stage larvae $\left(R^{2}=0.97, P \leq\right.$ $0.05)$, pupae $\left(R^{2}=0.97, P \leq 0.05\right)$ and adults $\left(R^{2}=0.97, P \leq 0.05\right)$.

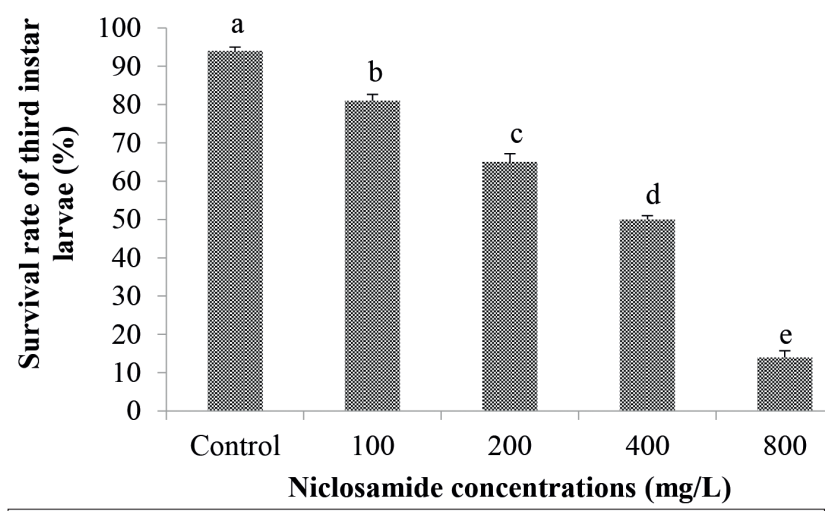

Figure 1. The effects of niclosamide on the survival rate of D. melanogaster $3^{\text {rd }}$ larval stage. Each columns represents the mean of four treatment groups, 25 insects in this developmental stages were used for each groups. The columns containing the same letter are not different from each other, $P \leq 0.05$ ( $X^{2}$ test). 
In the control culture, $94.00 \pm 1.00 \%$ of the larvae reached the third stage and this rate decreased to $14.00 \pm 1.73 \%$ at the highest niclosamide concentration of $800 \mathrm{mg} / \mathrm{L}$. Similarly, the survival rates decreased to $81.00 \pm 1.65,65.00 \pm 2.17$ and 50.00 \pm 1.00 at 100,200 and $400 \mathrm{mg} / \mathrm{L}$ niclosamide concentrations, respectively (Figure 1 ).

The rate of reaching the pupal stage in the control culture was $94.00 \pm 1.00$, while the rate of reaching the pupal stage decreased $81.00 \pm 1.65,63.00 \pm 3.57,50.00 \pm 1.00,14.00 \pm 1.73$ in niclosamide concentrations $(100,200,400,800 \mathrm{mg} / \mathrm{L})$, respectively (Figure 2$)$.

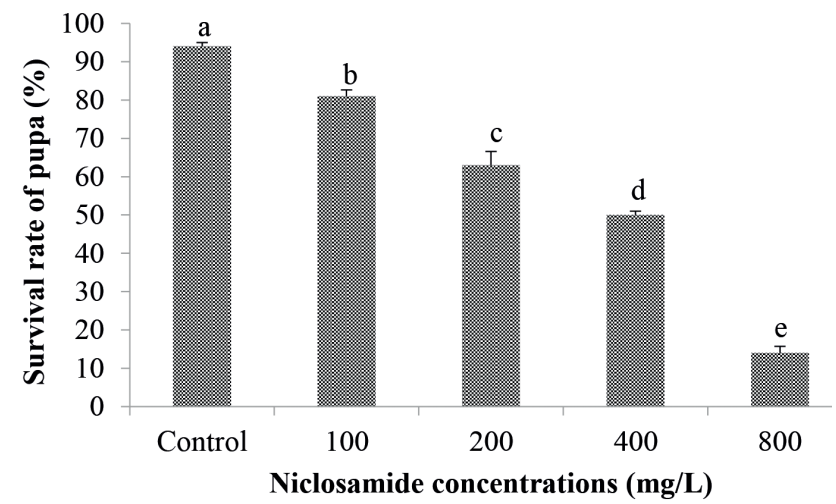

Figure 2. The effects of niclosamide on the survival rate of $D$. melanogaster pupa. Each columns represents the mean of four treatment groups, 25 insects in this developmental stages were used for each groups. The columns containing the same letter are not different from each other, $\mathrm{P} \leq 0.05$ ( $\mathrm{X}^{2}$ test).

Similar to the larval and pupal stage survival rates, the survival rate of the adult individuals decreased significantly when compared to the control culture. The survival rate significantly decreased at $800 \mathrm{mg} / \mathrm{L}$ niclosamide concentration, the highest concentration tested, to $11.00 \pm 0.86$, while in control culture, the survival rate was $94.00 \pm 1.00$ (Figure 3).

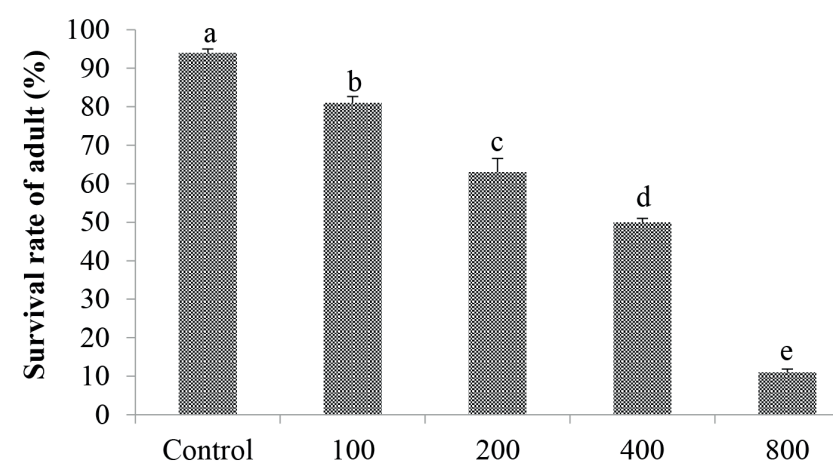

Niclosamide concentrations $(\mathrm{mg} / \mathrm{L})$

Figure 3. The effects of niclosamide on the survival rate of $D$. melanogaster adult. Each columns represents the mean of four treatment groups, 25 insects in this developmental stages were used for each groups. The columns containing the same letter are not different from each other, $\mathrm{P} \leq 0.05$ ( $\mathrm{X}^{2}$ test).
First three niclosamide concentrations $(100,200,400 \mathrm{mg} / \mathrm{L})$ did not have a statistically significant effect on the developmental time of $D$. melanogaster to reach the last larval stage, pupal and adult stages (Figures 4-6).

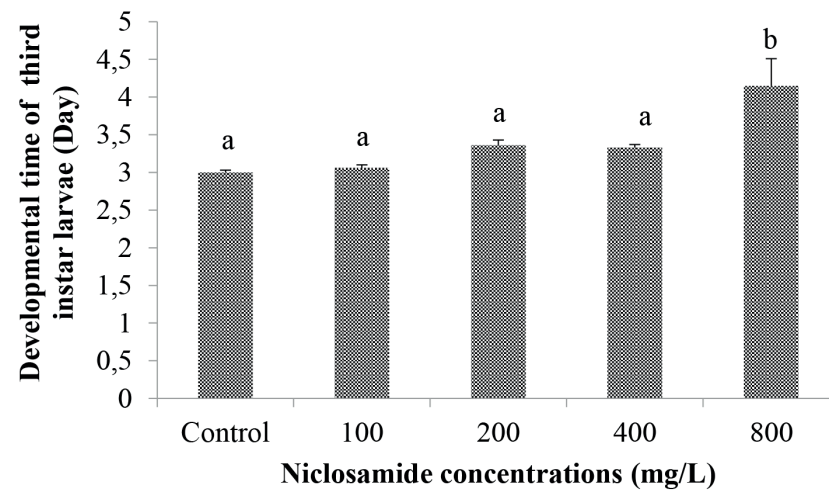

Figure 4. The effects of niclosamide on the developmental time of $D$. melanogaster $3^{\text {rd }}$ larval stage. Each columns represents the mean of four treatment groups, 25 insects in this developmental stages were used for each groups. The columns containing the same letter are not different from each other, $\mathrm{P} \leq 0.05$ (LSD test).

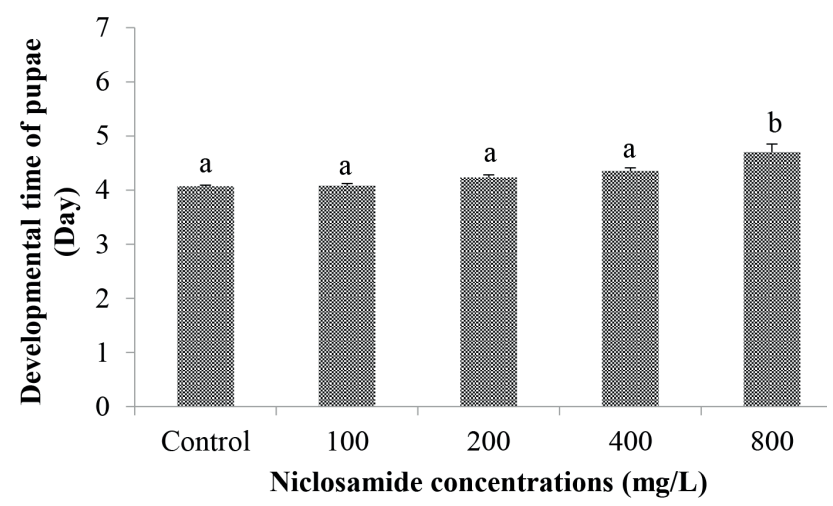

Figure 5. The effects of niclosamide on the developmental time of $D$. melanogaster pupa. Each columns represents the mean of four treatment groups, 25 insects in this developmental stages were used for each groups. The columns containing the same letter are not different from each other, $\mathrm{P} \leq 0.05$ (LSD test).

When the control culture was compared with the culture that contained the highest niclosamide concentration $(800 \mathrm{mg} / \mathrm{L})$, it was found that the highest tested niclosamide concentration prolonged the time required to reach the $3^{\text {rd }}$ larval to the pupal stages by 1 day and the time required to reach the adult stage was determined as 3 days. In the present study, we obtained a positive correlation between the tested concentrations and developmental time of larval $\left(R^{2}=0.88, P \leq 0.05\right)$, pupal $\left(R^{2}=\right.$ $0.93, P \leq 0.05)$, adult $\left(R^{2}=0.76 P \leq 0.05\right)$ stage. The developmental effects of niclosamide on $3^{\text {rd }}$ larval, pupal and adult individuals are presented in Figures 4-6. 


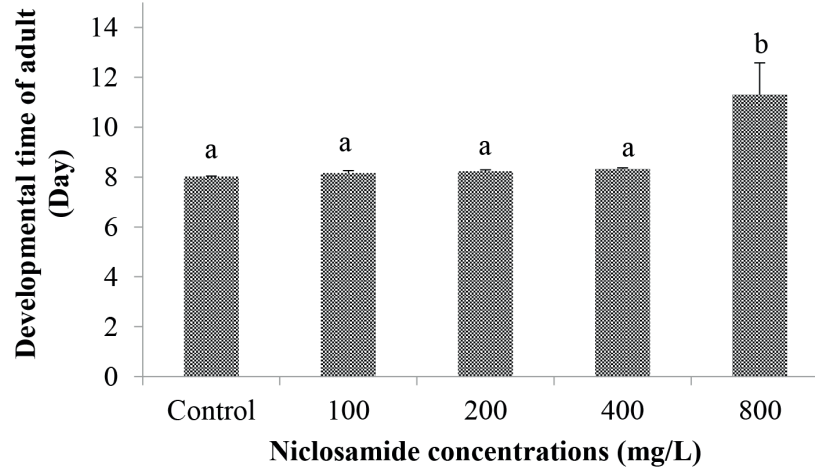

Figure 6. The effects of niclosamide on the developmental time of $D$. melanogaster adult. Each columns represents the mean of four treatment groups, 25 insects in this developmental stages were used for each groups. The columns containing the same letter are not different from each other, $\mathrm{P} \leq 0.05$ (LSD test).

\section{The Effects of Niclosamide on D. melanogaster Adult Longevity}

The effect of niclosamide on the adult longevity of adult $D$. melanogaster individuals is presented in Figure 7. The mean adult longevity was $42.08 \pm 0.50$ days in the control group, while it was $22.89 \pm 0.52$ days in $100 \mathrm{mg} / \mathrm{L}$ anthelmintic concentration, $16.35 \pm 0.33$ days in $200 \mathrm{mg} / \mathrm{L}$ concentration, $8.41 \pm 0.51$ days in $400 \mathrm{mg} / \mathrm{L}$ concentration, and $2.30 \pm 0.15$ days in $800 \mathrm{mg} / \mathrm{L}$ concentration. It was determined that the adult longevity decreased as the niclosamide concentration in the culture increased. These result was showed that a negative correlation was found between the tested niclosamide concentration and adult longevity of $D$. melanogaster $\left(R^{2}=0.096, P \leq 0.05\right)$

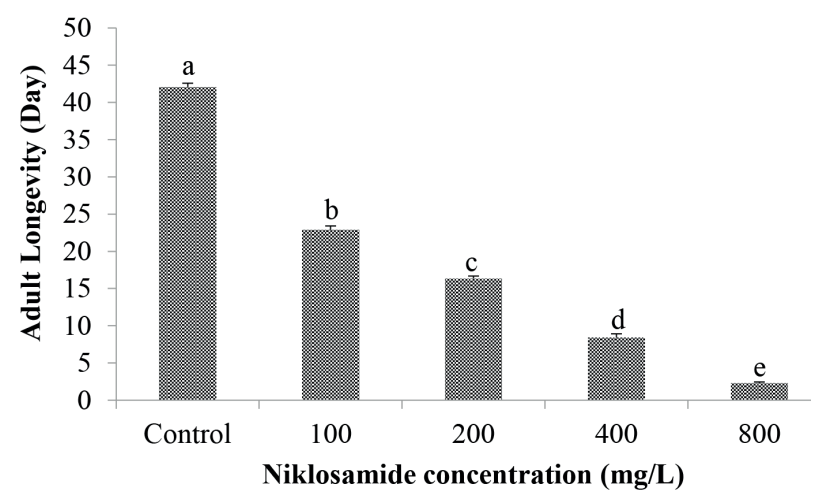

Figure 7. The effects of niclosamide on the adult longevity of $D$. melanogaster adult. Each columns represents the mean of four treatment groups, 25 insects in this developmental stages were used for each groups. The columns containing the same letter are not different from each other, $\mathrm{P} \leq 0.05$ (LSD test).

The Effects of Niclosamide on MDA, PCO, and GST Activity in Different Development Stages of D. melanogaster

The MDA levels in the $3^{\text {rd }}$ stage $D$. melanogaster larvae fed with artificial diets that included the tested niclosamide concentrations decreased when compared to the control

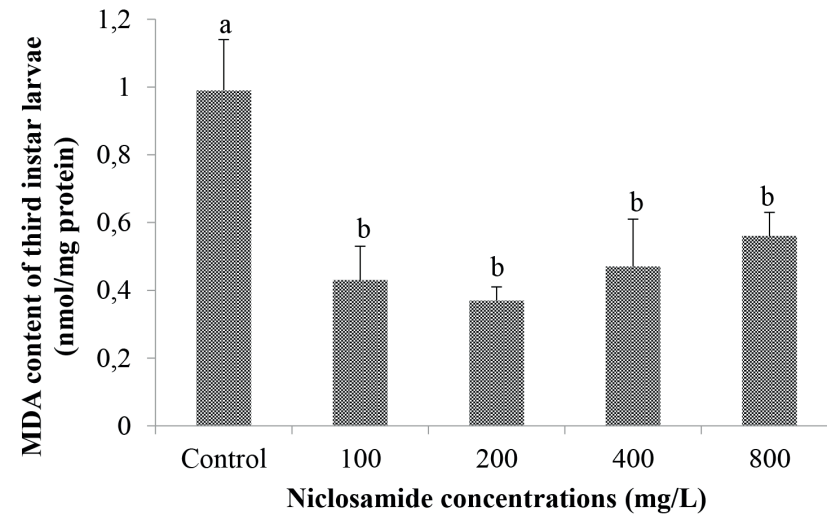

Figure 8. The effects of niclosamide on MDA levels in D. melanogaster $3^{\text {rd }}$ larval stage. Each columns represents the mean of four treatment groups, 25 insects in this developmental stages were used for each groups. The columns containing the same letter are not different from each other, $\mathrm{P} \leq 0.05$ (LSD test).

group. In the control group, the MDA level was $0.99 \pm 0.15$ $\mathrm{nmol} / \mathrm{mg}$ protein, while the MDA levels in all tested niclosamide concentrations $(100,200,400,800 \mathrm{mg} / \mathrm{L})$ were $0.43 \pm 0.10,0.37$ $\pm 0.04,0.47 \pm 0.14,0.56 \pm 0.07 \mathrm{nmol} / \mathrm{mg}$ protein, respectively (Figure 8). The MDA levels in the pupal stage insect cultured with different niclosamide concentrations decreased in 100, 200 and $800 \mathrm{mg} / \mathrm{L}$ concentrations when compared to the control group. The control group MDA level was $1.50 \pm 0.42 \mathrm{nmol} / \mathrm{mg}$ protein, while the MDA levels at all tested concentrations (100, $200,800 \mathrm{mg} / \mathrm{L}$ ) were $0.58 \pm 0.14,0.59 \pm 0.09,1.67 \pm 0.06$ and 0.71 $\pm 0.04 \mathrm{nmol} / \mathrm{mg}$ protein, respectively (Figure 9). Although we observed increasing MDA contents in adult stage insects, there was no statistically significant effect (Figure 10).

It was determined that the PCO content was $341.13 \pm 92.5$ $\mathrm{nmol} / \mathrm{mg}$ protein in the niclosamide-free control group $3^{\text {rd }}$ stage larvae. The PCO levels in the third stage $D$. melanogaster

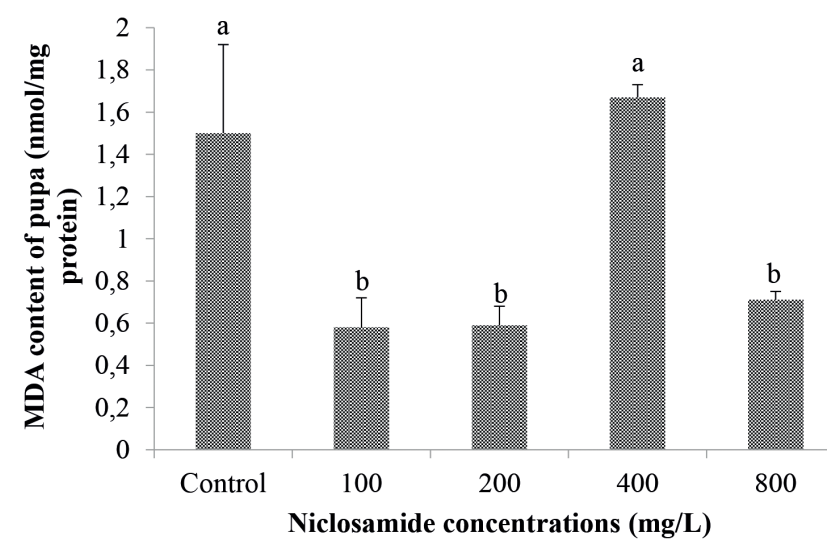

Figure 9. The effects of niclosamide on MDA levels in D. melanogaster pupa. Each columns represents the mean of four treatment groups, 25 insects in this developmental stages were used for each groups. The columns containing the same letter are not different from each other, $\mathrm{P} \leq 0.05$ (LSD test). 


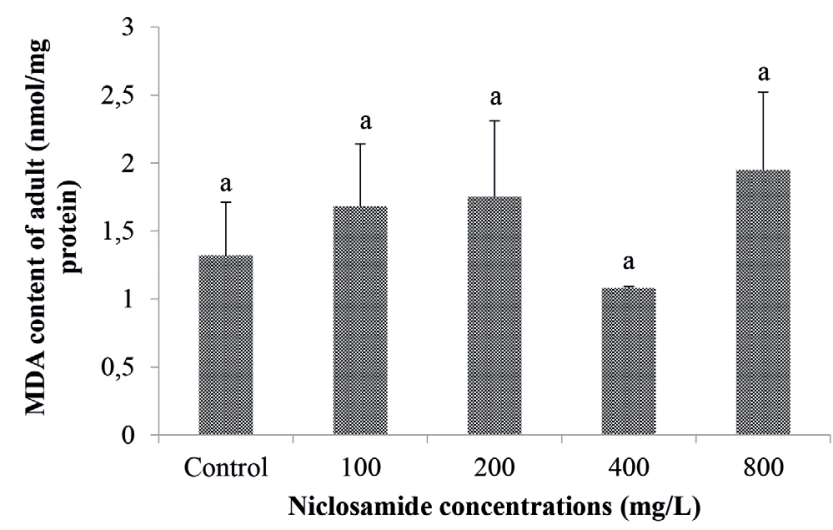

Figure 10. The effects of niclosamide on the MDA levels in $D$. melanogaster adult. Each columns represents the mean of four treatment groups, 25 insects in this developmental stages were used for each groups. The columns containing the same letter are not different from each other, $\mathrm{P} \leq 0.05$ (LSD test).

larvae in the groups cultured with artificial diets containing 100, 200 and $400 \mathrm{mg} / \mathrm{L}$ niclosamide concentrations were 97.68

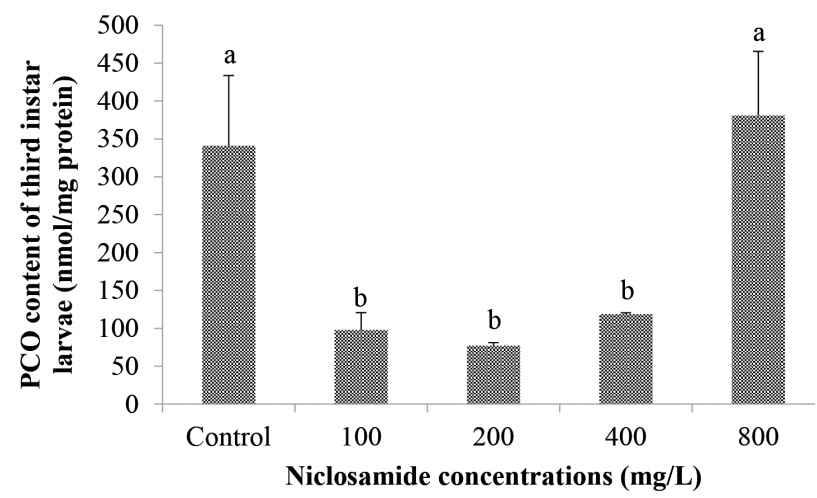

Figure 11. The effects of niclosamide on PCO levels in $D$. melanogaster $3^{\text {rd }}$ stage larvae. Each columns represents the mean of four treatment groups, 25 insects in this developmental stages were used for each groups. The columns containing the same letter are not different from each other, $\mathrm{P} \leq 0.05$ (LSD test).

$\pm 23.0,76.89 \pm 4.2,118.87 \pm 1.6 \mathrm{nmol} / \mathrm{mg}$ protein, respectively, and this decrease was statistically significant (Figure 11). Compared with the control group, there was no statistically effect in all the niclosamide groups, but our data showed that there was a significant effect between the $400 \mathrm{mg} / \mathrm{L}$ and 800 $\mathrm{mg} / \mathrm{L}$ niclosamide concentrations (Figure 12). In the control group, it was found that the PCO level in adult $D$. melanogaster individuals was $158.63 \pm 34.1 \mathrm{nmol} / \mathrm{mg}$ protein. Although the PCO level was $95.35 \pm 12.4 \mathrm{nmol} / \mathrm{mg}$ protein in the highest tested niclosamide concentration of $800 \mathrm{mg} / \mathrm{L}$, the difference was not statistically significant when compared to the control group. However, there was a significant effect difference between the highest niclosamide concentration and $200 \mathrm{mg} / \mathrm{L}$ niclosamide concentration (Figure 13).

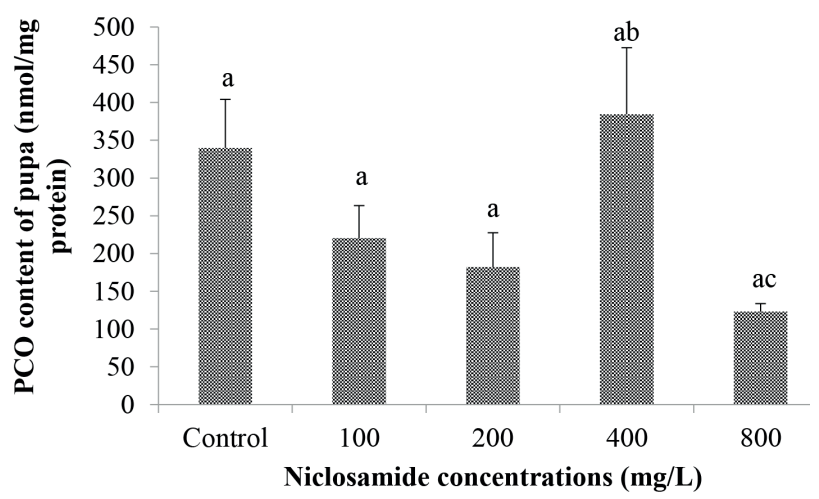

Figure 12. The effects of niclosamide on PCO levels in $D$. melanogaster pupa. Each columns represents the mean of four treatment groups, 25 insects in this developmental stages were used for each groups. The columns containing the same letter are not different from each other, $\mathrm{P} \leq 0.05$ (LSD test).

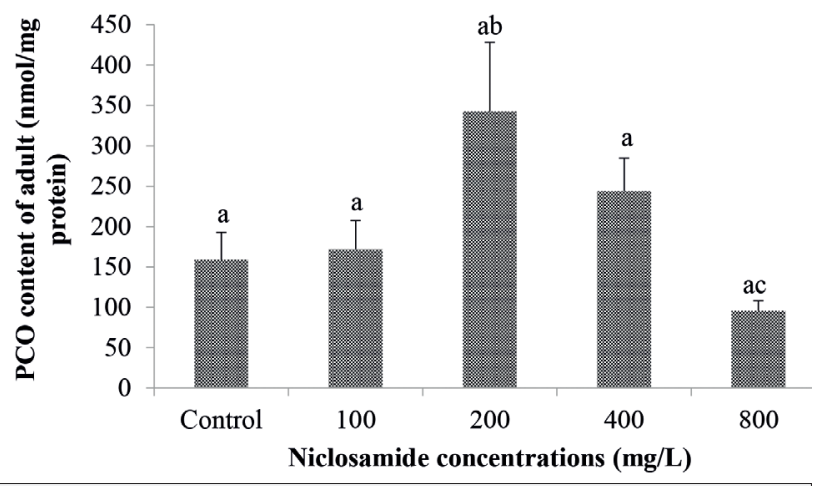

Figure 13. The effects of niclosamide on PCO levels in $D$. melanogaster adult. Each columns represents the mean of four treatment groups, 25 insects in this developmental stages were used for each groups. The columns containing the same letter are not different from each other, $\mathrm{P} \leq 0.05$ (LSD test).

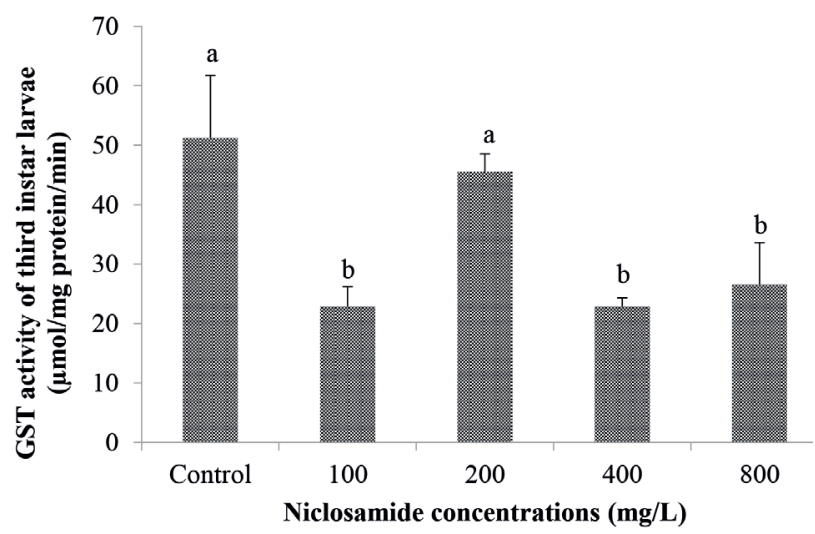

Figure 14. The effects of niclosamide on GST activity in D. melanogaster $3^{\text {rd }}$ stage larvae. Each columns represents the mean of four treatment groups, 25 insects in this developmental stages were used for each groups. The columns containing the same letter are not different from each other, $\mathrm{P} \leq 0.05$ (LSD test). 


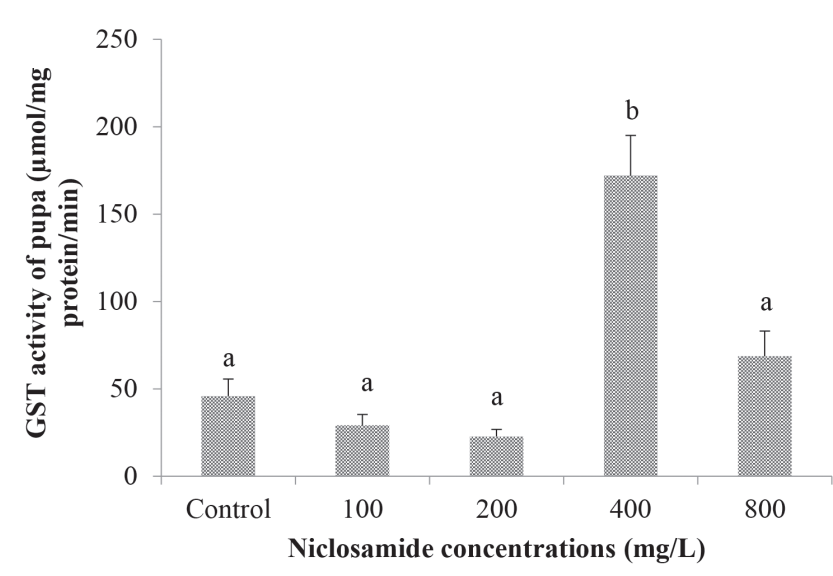

Figure 15. The effects of niclosamide on GST activity in D. melanogaster pupa. Each columns represents the mean of four treatment groups, 25 insects in this developmental stages were used for each groups. The columns containing the same letter are not different from each other, $\mathrm{P} \leq 0.05$ (LSD test).

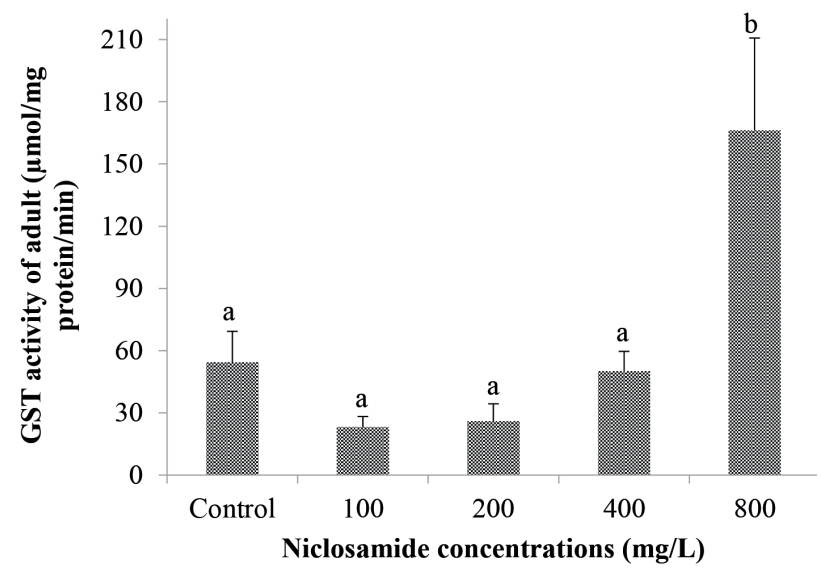

Figure 16. The effects of niclosamide on GST activity in D. melanogaster adult. Each columns represents the mean of four treatment groups, 25 insects in this developmental stages were used for each groups. The columns containing the same letter are not different from each other, $\mathrm{P} \leq 0.05$ (LSD test).

The effects of niclosamide on the GST activity in D. melonogaster larvae, pupae and adults were investigated in the present study conducted with the anthelmintic substance niclosamide, and it was observed that GST activity decreased at higher concentrations of niclosamide when compared to the control group results (Figures 14-16). Analysis of the impact of niclosamide on the GST activity in D. melanogaster pupa demonstrated that control group GST activity was $45.82 \pm 9.8 \mu \mathrm{mol} / \mathrm{mg}$ protein/min and GST activity significantly increased to $171.99 \pm 23.0 \mu \mathrm{mol} / \mathrm{mg}$ protein $/ \mathrm{min}$ at 400 $\mathrm{mg} / \mathrm{L}$ niclosamide concentration. It was observed that GST activity increased three-fold when the GST activity in the group with the highest niclosamide concentration $(800 \mathrm{mg} / \mathrm{L})$ was compared to that of the control group. The GST activity determined in the control group was $54.31 \pm 15.0 \mu \mathrm{mol} / \mathrm{mg}$ protein/min (Figure 15).

\section{DISCUSSION}

In the present study, the D. melanogaster model was used to research the effects of niclosamide, a salycilanide group anthelmintic substance, on vital parameters and antioxidant and oxidant levels of the insect. We investigated the effect of the niclosamide supplement to the semi-synthetic diet on the survival and development rates until adult stage and adult longevity of the newly hatched larvae of the model organism $D$. melanogaster. In the next stage, lipid peroxidation, protein oxidation and the antioxidant enzyme GST activity in different development stages of the insect (larval, pupal and adult stage) were investigated.

It was determined that all niclosamide concentrations significantly reduced the survival rate until the $3^{\text {rd }}$ larval, pupal and adult stages. In a study conducted by Sak and Uçkan (38) on the effects of cypermethrin on pupation and mortality rates of Galleria mellonella, and similar to our findings, it was determined that as the cypermethrin dose increased, larval development and pupation time were delayed, and, pupation ratio decreased. In certain studies, it was observed that administration of boronderivative chemicals such as boric acid and sodium tetraborate to various diets as pesticides delayed the development at high concentrations, decreased egg production and hatching, increased mortality in larval and pupal stages and adult longevity (39-43). In our study, the highest concentration delayed the development stage of the larvae, pupae, and adult stages of $D$. melanogaster and was also statistically significant. In the present study, a statistically significant decrease was observed in adult longevity with the increase in niclosamide concentration. It was determined that catalase (CAT) activity increased in Zaprionus paravittiger (Godbole \& Vaidya) fed with a diet that included antioxidant sodium hypophosphite, and there was a positive correlation between adult longevity and CAT activity (44). Similarly, the adult longevity of Z. paravittiger, fed with low concentrations of butylated hydroxyl anisole as an antioxidant, increased, its development was delayed, and egg production was decreased (45). The findings of the present study demonstrated that the adult longevity significantly decreased in the highest concentration of niclosamide when compared to the control culture, and the antioxidant enzyme GST activity increased significantly at the same concentration in the adult stage.

The highest niclosamide concentration $(800 \mathrm{mg} / \mathrm{L})$ led to a significant delay in all development stages, while other tested niclosamide concentrations did not have a significant impact on the developmental time. In a previous study by Büyükgüzel and Kayaoğlu (46), the in vivo insecticidal effect of niclosamide was investigated on G. mellonella larvae and it was determined that niclosamide significantly reduced the survival rates of $7^{\text {th }}$ stage larvae, pupae and adults, and similar to the present findings, the highest niclosamide concentration (1.0\%) significantly prolonged the adult developmental time. The highest niclosamide concentration delayed larval development by 5 days, pupal development by 4 days and adult stage by 6 days. In 
a similar study, excessive production of the peptides (peptide$\mathrm{S}$-methionine sulfoxide reductase) that contain methionine, an amino acid that plays a role in the treatment of oxidative damage, in $D$. melanogaster increased the maximum adult longevity, excessive production of glutamate-cysteine-ligase increased the adult longevity by $24 \%$, excessive production of apolipoprotein D was effective in the resistance against hydrogen peroxide $\left(\mathrm{H}_{2} \mathrm{O}_{2}\right)$ and prolonged the adult longevity of $D$. melanogaster by $26 \%$ (47). In a study conducted by Cruz et al. (48), boric acid that was added to the Apis mellifera (L.) culture at the rates of 2.5 and $7.5(\mathrm{mg} / \mathrm{g}$ diet) increased the mortality rate in worker larvae compared to the control group and $2.5 \mathrm{mg}$ boric acid administration killed $100 \%$ of the larvae on the $5^{\text {th }}$ and $6^{\text {th }}$ days, while the mortality rate with the $7.5 \mathrm{mg}$ dose was $100 \%$ on the $4^{\text {th }}$ day.

It is known that several biomolecules play a role in the regulation of various enzyme activities and preservation of the cell membrane integrity. Thus, the diet intake during the larval time has a significant impact on the development of the insect (49). The present study determined that niclosamide had a negative impact on the survival and development parameters of the insect and significant changes were observed in MDA, PCO levels and GST activity in all development stages. A similar effect was observed with the significantly increased MDA levels in the midgut of G. mellonella larvae that were cultured with streptomycin (11). It was determined that MDA and hence, GST activities significantly decreased in 100, 200, 400 and 800 $\mathrm{mg} / \mathrm{L}$ niclosamide supplementation to the insect larvae culture when compared to the control group. It was observed that niclosamide did not alter the structure of the culture medium where the insect was cultured, and even had a positive effect on the existing artificial diet components. Microbial contamination is the most common problem in insect species artificially cultured with artificial diets in a laboratory environment (10). Thus, various antimicrobial agents have been used in culture studies to prevent contaminations due to artificial culture media and to culture high quality adult individuals (12-14). Based on the present study findings, it is suggested that the tested niclosamide concentrations had a positive impact on the MDA, PCO levels and GST activities in the larval stage when the diet quality was increased by increasing the diet quality, and that a decrease in MDA levels during larval and pupal stages could be due to an adaptation developed by the insect. It can also be suggested that the reduction in PCO and MDA levels could be due to the proper functioning of the insect antioxidant defense system enzymes. Furthermore, it is known that the same insect species could require different physiological conditions and diets in different development stages (37). Thus, the changes in the effects of the tested niclosamide in the diet mixture on the life parameters and biochemical analyzes conducted in different stages were expected results.

It is known that xenobiotics lead to an increase in MDA and PCO levels due to the oxidative stress in tissues (50-53). It was determined that a $400 \mathrm{mg} / \mathrm{L}$ niclosamide concentration in the pupal stage and $200 \mathrm{mg} / \mathrm{L}$ concentration in the adult stage of $D$. melanogaster significantly increased PCO levels, one of the important oxidative stress markers. These findings demonstrated that the effects of niclosamide on the biological properties of insects were related to oxidative stress, and it also showed that these effects varied based on niclosamide concentration and the developmental stage of the insects. It was determined that GST activity significantly increased with $400 \mathrm{mg} / \mathrm{L}$ niclosamide concentration in pupal stage and with the highest concentration of $800 \mathrm{mg} / \mathrm{L}$ in the adult stage. It was observed that this increase was parallel to the increase in the PCO levels. The findings suggested that niclosamide could affect life parameters and other biochemical functions in insects through a second activity mechanism, namely the oxidative stress it causes, in addition to its primary activity. A similar effect was reported by Agianian et al. (54). They reported that two major forms of GST were found in D. melanogaster; GST-Delta (GST-1) and GST 2, and a third form of GST was found in Drosophila simulans (Sturtevant) (GST-Epsilon). Hunaiti et al. (55) found that the GST-Delta and the third form increased resistance to insecticides in Drosophila, while GST 2 was found in the flight muscles of the insect. In another study, it was demonstrated that 620 ppm and higher (1250 and 2500 ppm) concentrations of boric acid decreased the survival rate of $G$. mellonella, shortened the developmental time and extended the adult life span, and the same concentrations increased the MDA levels in the hemolymph and fat tissues of newborn individuals and significantly altered the antioxidant enzymes superoxide dismutase (SOD), CAT, GST and glutathione peroxidase (GPx) activities in final stage larvae and new pupae (43). A previous study investigated the effect of endosulfan on GST activity in different stages of Helicoverpa armigera (Hübner), and it was found that the activity was the lowest in insect eggs, pupae and adults, and it was suggested that this decrease was the resistance of the insect against the chemical exposure (56).

Several studies on certain insect species demonstrated that xenobiotics led to oxidative stress in various insect tissues and alterations were observed in antioxidative defense mechanisms against this stress $(32,57-60)$. The increase in GST activity in the pupal and adult stages of $D$. melanogaster in high niclosamide concentrations $(400-800 \mathrm{mg} / \mathrm{L})$ could be due to the prooxidant effect of niclosamide supplement in the culture. In a study conducted on the effects of different concentrations of diazinon, an organophosphate insecticide, on SOD and CAT enzyme activities in Pimpla turionellae (Linnaeus), it was reported that SOD activity significantly increased when compared to the control group, and as a result, CAT activity increased as well (61). The effects of pyriproxyfen, a juvenile hormone analog, on SOD and CAT activities in G. mellonella larvae was investigated and 0.0001, 0.0005, 0.001 and $0.005 \mathrm{mg} / \mathrm{ml}$ pyriproxyfen doses increased the SOD and CAT antioxidant enzyme activities after 24, 48 and 72 hours of application (62). Another study demonstrated that organophosphate insecticide fenitrothion induced changes in fatty tissue weight and SOD and CAT antioxidant enzyme activities in cotton moth Spodoptera exigua L. and flour pest Tenebrio molitor L. (63) Cadmium administration increased 
MDA levels, a lipid peroxidation product, in Oncopeltus fasciatus (Dallas), a heteropteran species, and reduced the activities of certain antioxidant enzymes (CAT, GR, GST) (64).

\section{CONCLUSION}

We believe that this study contributes to science in three ways. Firstly, there is no information regarding the effect of niclosamide on insects when added to the Drosophila diet under laboratory conditions. Secondly, our data showed that the low concentrations of niclosamide may be applied to prevent diet contamination in mass production of insects in laboratory conditions. Thirdly, the high concentrations of this chemical can negatively affect survival, development and certain physiological parameters of the insect. In this context, with the results obtained from this study the significance of $D$. melanogaster, which is an important model organism, in toxicology studies is revealed. Due to the above mentioned reasons, it can be concluded that this is a pioneering study in its evaluate as an alternative chemical substance that may be used in against to harmful insects. While various studies were conducted with antibiotics with different mechanisms of action and chemical structures on various insect groups, further detailed studies on a wider group of insects should also be conducted.

Peer-review: Externally peer-reviewed.

Author Contributions: Conception/Design of study: E.B.; Data Acquisition: G.Ü.; Data Analysis/Interpretation: E.B., G.Ü.; Final Approval and Accountability: E.B., K.B., G.Ü.; Drafting Manuscript: E.B., G.Ü.; Critical Revision of Manuscript: E.B., G.Ü.; Technical or Material Support: E.B., K.B., G.Ü.; Supervision: E.B.

Conflict of Interest: The author has no conflict of interest to declare.

Financial Disclosure: There are no funders to report for this submission

Acknowlegements: This study was supported by Research Fund of Zonguldak Bülent Ecevit University, Project No: 201550737594-01.

\section{REFERENCES}

1. Ozparlak $H$. The structure of cuticle, moulting and effects of diflubenzuron (DFB) in insects. J Sci Fac Art Sci. Selçuk Uni 2003; 21: 7-19.

2. Singh P, House HL. Antimicrobials safe levels in a synthetic diet of an insect, Agria affinis. J Insect Physiol 1970; 16: 1769-82.

3. Grenier S, Liu WH. Antifungals: Mold control and safe levels in artificial media for Trichogramma (Hymenoptera: Trichogrammatidae). Entomophaga 1990; 35: 283-91.

4. Pearson B, Raybould AF. The Effects of antibiotics on the development of larvae and the possible role of bacterial load in caste determination and diapause in Myrmicarubra (Hymenoptera: Formicidae). Sociobiol 1998; 31: 77-90.

5. Büyükgüzel K. DNA gyrase inhibitors: Novobiocin enhances the survial of Pimpla turionellae larvae reared on an arftifical diet but other antibiotics do not. J Appl Entomol 2001; 125: 583-87.
6. Büyükgüzel K, Yazgan Ş. Effects of antimicrobial agents on survival and development of larvae of Pimpla turionellae L. (Hymenoptera: Ichneumonidae) reared on an artificial diet. Turk J Zool 2002; 26: 111-19.

7. Alverson J, Cohen AC. Effect of antifungal agents on biological fitness of Lygus hesperus (Heteroptera: Miridae). J Econ Entomol 2002; 65: 256-60.

8. Inglis GD, Cohen AC. Influence of antimicrobial agents on the spoilage of a meat-based entomophage diet. J Econ Entomol 2004; 97: 235-250.

9. Büyükgüzel $E$, Kalender Y. Penicillin-Induced Oxidative Stress: Effects on Antioxidative Response of Midgut Tissues in Larval Instars of G. mellonella. J Econ Entomol 2007; 100: 1533-41.

10. Büyükgüzel $E$, Kalender Y. Galleria mellonella survivorship, development and protein content in response to dietary antibiotics. J Entomol Sci 2008; 43: 27-40.

11. Büyükgüzel $E$, Kalender $Y$. Exposure to streptomycin alters oxidative and Antioxidative response in larval midgut tissues of Galleria mellonella. Pestic Biochem Physiol 2009; 94: 112-18.

12. Büyükgüzel $E$, Büyükgüzel K. Effect of acyclovir on the microbial contamination in the artifical and natural diets for rearing of Galleria mellonella L. Karaelmas Sci Eng J 2016a; 6: 105-10.

13. Büyükgüzel $E$, Büyükgüzel $K$. Effects of antiviral agent, acyclovir, on the biological fitness of Galleria mellonella (Lepidoptera: Pyralidae) adults. J Econ Entomol 2016b; 109: 2090-95.

14. Çalık G, Büyükgüzel $K$, Büyükgüzel $E$. Reduced fitness in adults from larval, Galleria mellonella (Lepidoptera: Pyralidae) reared on media amended with the anthelmintic, mebendazole. J Econ Entomol 2016; 109: 1-6.

15. Büyükgüzel $E$, Erdem $M$, Tunaz $H$, Küçük $C$, Atılgan UC, Stanley $D$, et al. Inhibition of eicosanoid signaling leads to increased lipid peroxidation in a host/parasitoid system. Comp Biochem Physiol Part A: Mol Integ Physiol 2017; 204: 121-28.

16. Kelly J, Kavanagh K. Caspofungi.n primes the immune response of the larvae of Galleria mellonella and induces a non-specific antimicrobial response. J Med Microbiol 2011; 60: 189-96.

17. Ashburner M. Drosophila a Laboratory Handbook, New York: Cold Spring Harbor Press; 1989.

18. Tennessen, J M, Barry, WE, Cox, J, Thummel, CS. Methods for studying metabolism in Drosophila. Methods 2014; 68: 105-15.

19. Çakır Ş, Sarıkaya R. Effects of some organophosphate insecticides on percentage of survival of Drosophila melanogaster. J Gazi Educ Fac 2004; 24: 71-80.

20. Sarıkaya R, Selvi M, Akkaya N, Acar M, Erkoç F. Effects of food dyes in different concentrations on percentage of survival in Drosophila melanogaster (mwh x flr), SDU J Sci (E- Journal) 2010; 5: 38-46.

21. Zhou J, Jin B, Jin Y, Liu Y, Pan J. The antihelminthic drug niclosamide effectively inhibits the malignant phenotypes of uveal melanoma in vitro and in vivo. Theranostics 2017; 7: 1447-62.

22. Öztop $A Y$, Saygı G, Öztop HN. In vitro effects of some anthelminthics on the malate dehydrogenase and lactate dehydrogenase enzyme activities of Taenia Saginata. Tr J Med Sci 1999; 29: 365-70.

23. Şanlı Y, Kaya S. Veteriner Farmakoloji ve Illaçla Sağıtım Seçenekleri kitabı, 2.Baskı. Ankara: Medisan Yayınevi; 1994.p.651-69.

24. Lobo, V, Patil, A, Phatak, A, Chandra, N. Free radicals, antioxidants and functional foods: Impact on human health. Pharmacognosy reviews 4(8), 118-26.

25. Petersen, RC. Free-radicals and advanced chemistries involved in cell membrane organization influence oxygen diffusion and pathology treatment. AIMS Biophysics, 2017; 4: 240-83. 
26. Birben, E., Sahiner, U M., Sackesen, C, Erzurum, S, Kalayci, O. (2012). Oxidative stress and antioxidant defense. WAO Journal, 2010; 51: 9-19.

27. Zhu, YC, Gordon, LS, Ming,SC. Comparative study on glutathione S-transferase activity, cDNA, and gene expression between malathion susceptible and resistant strains of the tarnished plant bug, Lygus lineolaris. Pest. Biochem. Physiol 2007; 87: 62-72.

28. Rogina B, Reenan RA, Nilsen SP, Helfand SL. Helfand, Extended lifespan conferred by contransporter gene mutations in Drosophila. Biogerontol Sci 2000; 290: 2137-40.

29. Lesch C, Goto A, Lindgren M, Bidla G, Dushay MS, Theopold U. A role for Hemolectin in coagulation and immunity in Drosophila melonagaster. Develop Com Immun 2007; 31: 1255-63.

30. Jain SK, Levine SN. Levine, Elevated lipid peroxidation and vitamin e-quinone levels in heart ventricles of streptozotocin-treated diabetic rats. Free Rad Biol Med 18: 337-41.

31. Levine RL, Williams JA, Stadtman ER., Shacter E. Carbonyl assays for determination of oxidatively modified proteins. Meth Enzymol 1994; 233: 346-57.

32. Krishnan N, Kodrík D. Antioxidant enzymes in Spodoptera littoralis (Boisduval): Are they enhanced to protect gut tissues during oxidative stress? J Insect Physiol 2006; 52: 11-20.

33. Habig HW, Pabst MJ, Jakoby WB. Glutathione-S-transferases: the first enzymatic step in mercapturic acid formation. J Biol Chem 1974; 249: 7130-39.

34. Lowry OH, Rosebrough NL, Farr AL. Randall RJ. Protein measurement with the folin phenol reagent. J Biol Chem 1951; 19: 265.

35. Pajot P. Fluorescence of proteins in 6-M Guanidine Hydrochloride. Euro J Biochem 1976; 63: 263-69.

36. Snedecor GS, Cochran WG. Cochran, Statistical methods, $8^{\text {th }}$ edn., lowa State University Press Ames IA 1989.

37. Grenier S, Delobel B, Bannot G. Physiological considerations of importance to the success of in vitro culture. J Insect Physiol 1986; 32: 403-8.

38. Sak O, Uçkan F. Effects of cypermethrin exposed to host on the developmental biology of Pimpla turionellae (Hymenoptera: Ichneumonidae). Ann Entomol Soc Am 2009; 102: 288-94.

39. Sak O, Uçkan F. Boric acid dust as a component of an integrated cockroach management program in confined swine production. J Econ Entomol 2003; 96: 1362-66.

40. Xue RD, Barnard DR. Barnard, Boric acid bait kills adult mosquitoes (Diptera: Culicidae). J Econ Entomol 2003; 96: 1559-62.

41. Gore JC, Schal C. Laboratory evaluation of boric acid-sugar solutions as baits for management of German cockroach infestiation. J Econ Entomol 2004; 97: 581-87.

42. Gore JC, Zurek L, Santangelo RG, Stringham MS, Watson DW, Schal C. Water solutions of boric acid and sugar for management of German cocroach populations in livestock production system. J Econ Entomol 2004; 97: 715-20.

43. Hyršl $P$, Büyükgüzel $E$, Büyükgüzel $K$. The effects of boric acidinduced oxidative stress on antioxidant enzymes and survivorship in Galleria mellonella. Arch Insect Biochem Physiol 2007; 66: 23-31.

44. Wadhwa R, Sharma SP. Studies on catalase in ageing Zaprionus paravittiger (Diptera) with special reference to an antioxidant feding. Mech Ageing Dev 1987; 40: 139-47.

45. Bains JS, Stephane HR, Baimoukhametova DV, Piet R. Retrograde regulation of GABA transmission by the tonic release of oxytocin and endocannabinoids governs postsynaptic firing. J Neurosci 2007; 27: 1325-33.

46. Büyükgüzel $E$, Kayaoğlu $S$. The effect of niclosamide on some biological and physiological aspects of Galleria mellonella L. (Lepidoptera: Pyralidae). Turk J Entomol 2014; 38: 83-99.
47. Muller FL, Lustgarten MS, Jang Y, Richardson A, Remmen HV. Trends in oxidative aging theories. Free Rad Biol Medic 2007; 43: 477-503.

48. Cruz SA, Silva ECM, Bueno OC, Malaspina O. Malaspina, Morphological alterations induced by boric acid and fipronil in the midgut of worker honey bee (Apis mellifera) larvae. Cell Biol Tox 2009; 26: 165-76.

49. Şahin N, Türkoğlu Ş. The effects of some textile dyes on the percentage of survival, longevity and number of offspring individuals of Drosophila melanogaster Cumhuriyet Un Fac Sci J 2014; 35: 1300-1949.

50. Wu KC, Liu J, Klaassen CD. Role of Nrf2 in preventing ethanol induced oxidative stress and lipid accumulation. Toxicol Appl Pharmacol 2012; 262: 321-29.

51. Erdem M, Büyükgüzel E. The Effects of Xanthotoxın on the biology and biochemistry of Galleria mellonella L. (Lepidoptera: Pyralidae). Arch Insect Biochem Physiol 2015; 89: 193-203.

52. Büyükgüzel E, Erdem M, Tunaz $H$, Küçük $C$, Atılgan UC, Stanley $D$, et al. Inhibition of eicosanoid signaling leads to increased lipid peroxidation in a host/parasitoid system. Comp Biochem Physiol Part A: Mol Integ Physiol 2017; 204: 121-28.

53. Güneş $E$, Büyükgüzel $E$. Oxidative effects of boric acid on different developmental stages of Drosophila melanogaster meigen (Diptera: Drosophilidae). Turk J Entomol 2017; 41: 3-15.

54. Agianian B, Tucker PA, Schouten A, Leonard K, Bullard B, Gros P. GrosStructure of a Drosophila Sigma class Glutathione S-transferase reveals a novel active site topography suited for lipid peroxidation products. J Mol Biol 2003; 326: 151-65.

55. Hunaiti AA, Elbettieha AM. Developmental studies on Drosophila melanogaster Glutathione -S-transferase and its induction by oxadiazolone. Insect Biochem Mol Biol 1995; 25: 1115-119.

56. Rajurkar RB, Khan ZH, Gujar GT. Studies on levels of Glutathione-STransferase, its isolation and purification from Helicoverpa armigera. Current Sci 2003; 85: 1355-60.

57. Slepneva IA, Glupov VV, Sergeeya SV, Khramtsov VV. Detection of reactive oxygen species in hemolymph of Galleria mellonella and Dendrolimus superans sibiricus (Lepidoptera) larvae. Biochem Biophys Res Commun 1999; 264: 215-5.

58. Glupov VV, Khyosheyskaya MF, Lozinskaya YL, Dubovski IM, Martemyanov VV, Sokolova JY. Application of the nitroblue tetrazolium-reduction method for studies on the production of reactive oxygen species in insect haemocytes. Cytobios 2001; 106: 165-78.

59. Lozinskaya YL, Slepneva IA, Khramtsov VV, Glupov VV. Changes of the antioxidant status and system of generation of free radicals in hemolymph of Galleria mellonella Larvae at Microsporidiosis. J Evol Biochem Phsycol 2004; 40: 119-25.

60. Aucoin JS, Jiang P, Aznavour N, Tong XK, Buttini M, Descarries L, et al. Selective cholinergic denervation, independent from oxidative stress, in a mouse model of Alzheimer's disease. Neurosci 2005; 132: $73-86$.

61. Kayış T, Emre I, Coşkun M. Effects of diazinon on antioxidant enzymes and adult emergence of the parasitoid Pimpla turionellae L. (Hymenoptera: Ichneumonidae). Turk J Entomol 2012; 36: 46371.

62. Sezer B, Ozalp P. Effect of juvenile hormone analogue, pyriproxyfen on antioxidant enzymes of greater wax moth, Galleria mellonella (Lepidoptera: Pyralidae: Galleriinae) Larvae. Pak J Zool 2015; 47: 665-69. 
Eur J Biol 2019; 78(1): 29-39

Ustundag et al. Oxidative Effect of Niclosamide on D. melanogaster

63. Adamski Z, Ziemnicki K, Fila K, Zikic RV, Stajn A. Stajn, Effects of long-term exposure to fenitrothion on Spodoptera exigua and Tenebrio molitor larval development and antioxidant enzyme activity. Biol Lett 2003; 40: 43-52
64. Cervera A, Maymo AC, Pardo RM, Garcera MD. Antiooxidant enzymes in Oncopeltus fasciatus (Heteroptera: Lygaeidae) exposed to cadmium. Environ Entomol 2003; 32: 705-10. 